\title{
Experiências vividas por famílias e crianças com sintomas urinários e intestinais: revisão sistemática de métodos mistos
}

\author{
Lived experiences by families and children with urinary and intestinal symptoms: systematic review of \\ mixed methods \\ Experiencias vividas por familias y niños con síntomas urinarios e intestinales: revisión sistemática de \\ métodos mixtos
}

\author{
Cristiane Feitosa Salviano ${ }^{1}$ (1) \\ Priscilla Lemos Gomes ${ }^{1}$ (D) \\ Gisele Martins ${ }^{1}$ (D)
}

1. Universidade de Brasília, Faculdade de

Ciências da Saúde, Brasília, DF, Brasil
AUTOR CORRESPONDENTE

Cristiane Feitosa Salviano

E-mail: crisenf.salviano@gmail.com

\section{Recebido em 05/05/2019.}

Aprovado em 06/01/2020.

\section{RESUMO}

Objetivo: Mapear e descrever a ocorrência de sintomas urinários e intestinais durante a infância e investigar o impacto de tais sintomas nas experiências vividas por crianças e suas famílias. Método: Revisão sistemática de métodos mistos realizada nas bases eletrônicas MEDLINE/PUBMED, CINAHL, LILACS, PSYCINFO e EMBASE em julho de 2019, as quais geraram 3.020 referências. Após remoção das duplicatas, 2.521 títulos e resumos foram triados com filtro de tempo, e aplicado critérios de inclusão. Desses, 31 artigos foram lidos na íntegra e avaliados quanto à qualidade metodológica pelo Mixed Methods Appraisal Tool, resultando em 15 artigos como amostra final. Resultados: Foram encontrados: sentimento de inferioridade, agressividade, culpa e vergonha. A revisão evidenciou, ainda, o impacto negativo dos sintomas urinários e/ou intestinais no contexto social da criança e de sua família, em especial, no ambiente escolar. Conclusão e implicações para a prática: Essa revisão sistemática de métodos mistos evidencia a importância de trabalhar os impactos emocionais e sociais da criança, em especial os eventos no ambiente escolar. Se faz necessário subsidiar o profissional de saúde na assistência às famílias e crianças com sintomas urinários e/ou intestinais, no sentido de prover um cuidado ampliado, valorizando as necessidades biopsicoemocionais da díade criança-família.

Palavras-chave: Revisão sistemática; Sintomas do Trato Urinário Inferior; Constipação; Saúde da Criança.

\begin{abstract}
Objective: To map and describe the occurrence of urinary and intestinal symptoms during childhood and to investigate the impact of such symptoms on the experiences of children and their families. Method: Systematic review of mixed methods, performed in the electronic databases MEDLINE/PUBMED, CINAHL, LILACS, PSYCINFO and EMBASE in July 2019, which generated 3,020 references. After removal of duplicates, 2,521 titles and abstracts were screened with time filter, and application of inclusion criteria. Among these, 31 articles were read in full and evaluated as for methodological quality by the Mixed Methods Appraisal Tool, resulting in 15 articles as the final sample. Results: The following results were found: feeling of inferiority, aggressiveness, guilt and shame. The review also showed the negative impact of urinary and/or intestinal symptoms in the social context of children and their families, especially in the school environment. Conclusion and Implications for practice: This systematic review of mixed methods highlights the importance of addressing children's emotional and social impacts, especially events in the school environment. It is necessary to subsidize the health professional in assisting families and children with urinary and/or intestinal symptoms, in order to provide expanded care, valuing the biopsychosocial needs of the child-family dyad.
\end{abstract}

Keywords: Systematic review; Lower Urinary Tract Symptoms; Constipation; Child Health.

\section{RESUMEN}

Objetivo: Mapear y describir la ocurrencia de síntomas urinarios e intestinales durante la infancia e investigar su impacto en las experiencias de los niños y sus familias. Método: Revisión sistemática de métodos mixtos, realizada en las bases de datos electrónicas MEDLINE/PUBMED, CINAHL, LILACS, PSYCINFO, EMBASE en julio de 2019, las cuales generaron 3,020 referencias. Después de eliminar los duplicados, se seleccionaron 2.521 títulos y resúmenes con filtro de tiempo, y se aplicaron criterios de inclusión. De esos, 31 artículos fueron totalmente leídos y evaluados en cuanto a la calidad metodológica por Mixed Methods Appraisal Tool, resultando en 15 artículos como muestra final. Resultados: Fueron encontrados: sentimiento de inferioridad, agresividad, culpa y vergüenza. También se notó el impacto negativo de los síntomas urinarios y/o intestinales en el contexto social de los niños y sus familias, especialmente en el escolar. Conclusión e implicaciones para la práctica:: Esta revisión sistemática resalta la importancia de abordar los impactos emocionales y sociales de los niños, especialmente en la escuela. Se necesita subsidiar el profesional de salud en la atención a las familias y niños con síntomas urinarios y/o intestinales, a fin de proporcionar un cuidado ampliado, valorando las necesidades biopsicosociales de la díada niño-familia.

Palabras clave: Revisión sistemática; Síntomas del Tracto Urinario Inferior; estreñimiento; Salud del Niño. 


\section{INTRODUÇÃO}

O diagnóstico clínico de sintomas de trato urinário inferior e intestinal é realizado por meio de anamnese, exame físico e exames complementares (urofluxometrias, ultrassom de rins e vias urinárias, exame de urina tipo 1). ${ }^{1,2}$ Adicionalmente, recomenda-se a aplicação de diários de eliminações, bem como escalas validadas para detecção e mensuração de sintomas urinários (ex. Dysfuncional Voiding Score System ${ }^{3}$ ), além de escalas visuais como a escala fecal de Bristol para identificação do aspecto e consistência fecal. ${ }^{1,4}$ A coleta dessa história clínica baseada na avaliação física e relato da criança e de sua família se vale, dessa forma, de dados objetivos e subjetivos.

A combinação de dados (objetivos e subjetivos) permite uma percepção ampliada da condição clínica do paciente. A experiência de sintomas é, portanto, compreendida quando o profissional consegue captar outros aspectos que não apenas os sintomas e condições descritos em normatizações diagnósticas. Assim, os antecedentes pessoais, as emoções desencadeadas, os desconfortos, as consequências no cotidiano são exemplos de elementos que constituem o referencial teórico descrito por Lenz et al. ${ }^{5}$ sobre experiência de sintomas desagradáveis.

Ante o exposto, justifica-se a necessidade de realizar uma busca mais ampliada desses demais elementos objetivos e subjetivos para uma compreensão mais multifacetada sobre a ocorrência e experiência de sintomas urinários e intestinais na infância, a fim subsidiar o profissional de saúde no atendimento às crianças acometida por esses sintomas. O conhecimento ampliado desses outros fatores associados a ocorrência de sintomas urinários e intestinais durante a infância pode expandir a percepção do profissional de saúde, potencializando sua avaliação, a fim de captar a experiência da criança e da família, aproximando-se de dimensões não apenas biológicas. ${ }^{6}$ Desse modo, o profissional é capaz de prover um cuidado individualizado e focado nos sintomas e problemas que causam maior incômodo à criança. Consequentemente, a aceitação e adesão ao plano terapêutico embasado em metas traçadas em conjunto com a criança e família poderão ser mais facilmente implementadas e alcançadas, tais como as intervenções de uroterapia. ${ }^{1,7}$

Desta forma, este estudo visou mapear e descrever a ocorrência de sintomas urinários e intestinais durante a infância e investigar o impacto de tais sintomas nas experiências vividas por crianças e suas famílias.

\section{MÉTODO}

O presente estudo se trata de uma Revisão Sistemática de Métodos Mistos, a qual é definida como um tipo de revisão da literatura cujo objetivo é identificar, selecionar, avaliar e sintetizar estudos quantitativos, qualitativos e de métodos mistos. Desse modo, tal revisão oferece uma síntese do conhecimento produzido, facilitando o processo de tomada de decisão dos profissionais, baseada em evidências. ${ }^{8}$

Esse tipo de revisão segue sete etapas: ${ }^{8}$ 1) delimitar da pergunta norteadora da revisão (ou perguntas qualitativas e quantitativas); 2) definir dos critérios de inclusão e exclusão; 3) realizar busca extensiva da literatura; 4) identificar potenciais estudos por meio de avaliação de título e resumo; 5) Selecionar artigos com base no texto completo; 6) avaliar a qualidade dos estudos inclusos; 7) Sintetizar os estudos inclusos.

Tendo em vista a primeira etapa desta revisão, tem-se a seguinte pergunta norteadora: Quais são os sinais, sintomas e problemas relacionados à disfunção vesical e intestinal na infância, relatados ou experienciados/vividos por crianças e pais/ responsáveis?

A busca foi realizada em julho de 2019, nas bases eletrônicas Medical Analysis and Retrievel System Online (MEDLINE/PubMed), Cumulative Index to Nursing and Allied Health Literature (CINAHL), Literatura Latino-Americana e do Caribe em Ciências da Saúde (LILACS), American Psychological Association (PsycINFO) e Excerpta Medica dataBASE (EMBASE). A estratégia de busca utilizou descritores obtidos no Medical Subject Headings (MeSH), cuja combinação foi mediada pelos boleanos OR e AND, conforme representado na Figura 1.

Teve-se como critérios de inclusão para a amostra: crianças e adolescentes na faixa etária de 6 a 18 anos (faixa etária delimitada com base no conceito de DVI que aponta a ocorrência de sintomas em crianças acima de 5 anos); ${ }^{1}$ abordar a percepção e/ou experiências vividas pelas famílias bem como pelas crianças sobre os sintomas de DVI; estudos com delineamento qualitativos, quantitativos e de métodos mistos; artigos publicados nos idiomas português, inglês e espanhol; publicados nos últimos 10 anos (2009 a 2019). Enquanto que como critérios de exclusão foram adotados: estudos realizados com crianças e/ou pais e famílias de crianças com problemas neurológicos ou com alterações cognitivas/desenvolvimentais.

A busca nas bases de dados gerou 3020 referências. A seleção dos estudos foi conduzida por meio de exportação dos resultados das buscas nas bases eletrônicas para o gerenciador de referências EndNote versão X8. Com o programa, foram removidas 499 duplicatas e 1.205 no quesito de temporalidade (últimos 10 anos) resultando em 1.316 para avaliação dos demais critérios de inclusão por meio da leitura de títulos e resumos, esta foi realizado com a participação de dois revisores independentes. Após avaliação de elegibilidade, 31 artigos apresentaram potencial de inclusão na amostra final e, dentre esses, 15 foram escolhidos para a amostra final após a leitura na íntegra (Figura 2).

A Avaliação da qualidade metodológica dos artigos incluídos foi realizada com o instrumento MMAT (Mixed Methods Appraisal Tool - Versão 2011). Tal instrumento foi proposto por Pluye \& Hong, ${ }^{8}$ sendo que o MMAT tem como objetivo permitir a avaliação simultânea de estudos que empregam diferentes metodologias tais como qualitativa, quantitativa e método misto. ${ }^{8}$ A ferramenta é composta de um conjunto de 20 critérios de qualidade distribuídos em cinco tipos metodológicos (estudos qualitativos, quantitativos - clínicos randomizados, quantitativos não randomizados, quantitativo descritivo, estudos mistos). A depender do tipo de estudo foram avaliados os respectivos 


\begin{tabular}{|c|}
\hline "lower urinary tract \\
symptoms" \\
ORconstipationOR "bladder \\
diseases"OR "urinary \\
retention"OR enuresisOR \\
encopresis OR "bladder and \\
bowel dysfunction" OR \\
bedwetting OR "daytime \\
wetting" OR "dysfunctional \\
voiding" OR "voiding \\
dysfunction" OR "daytime \\
incontinence" OR "nocturnal \\
enuresis" \\
\hline
\end{tabular}
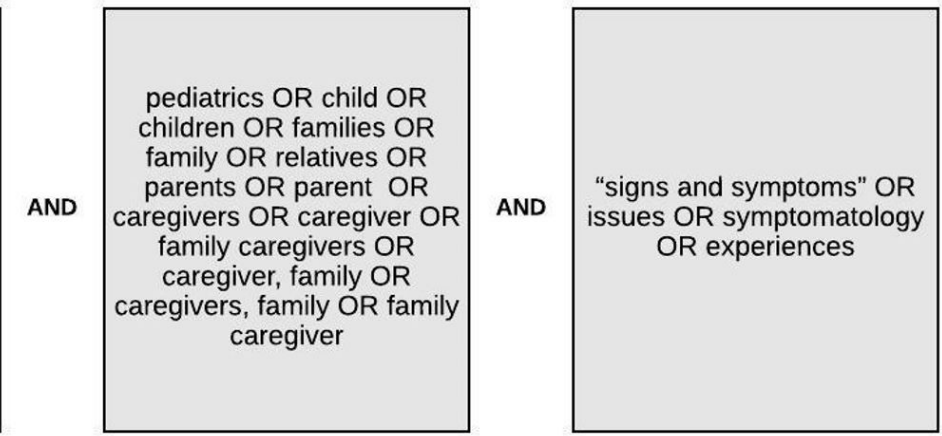

Figura 1. Estratégia de busca por meio da combinação de descritores. Brasília/DF, Brasil, 2019.

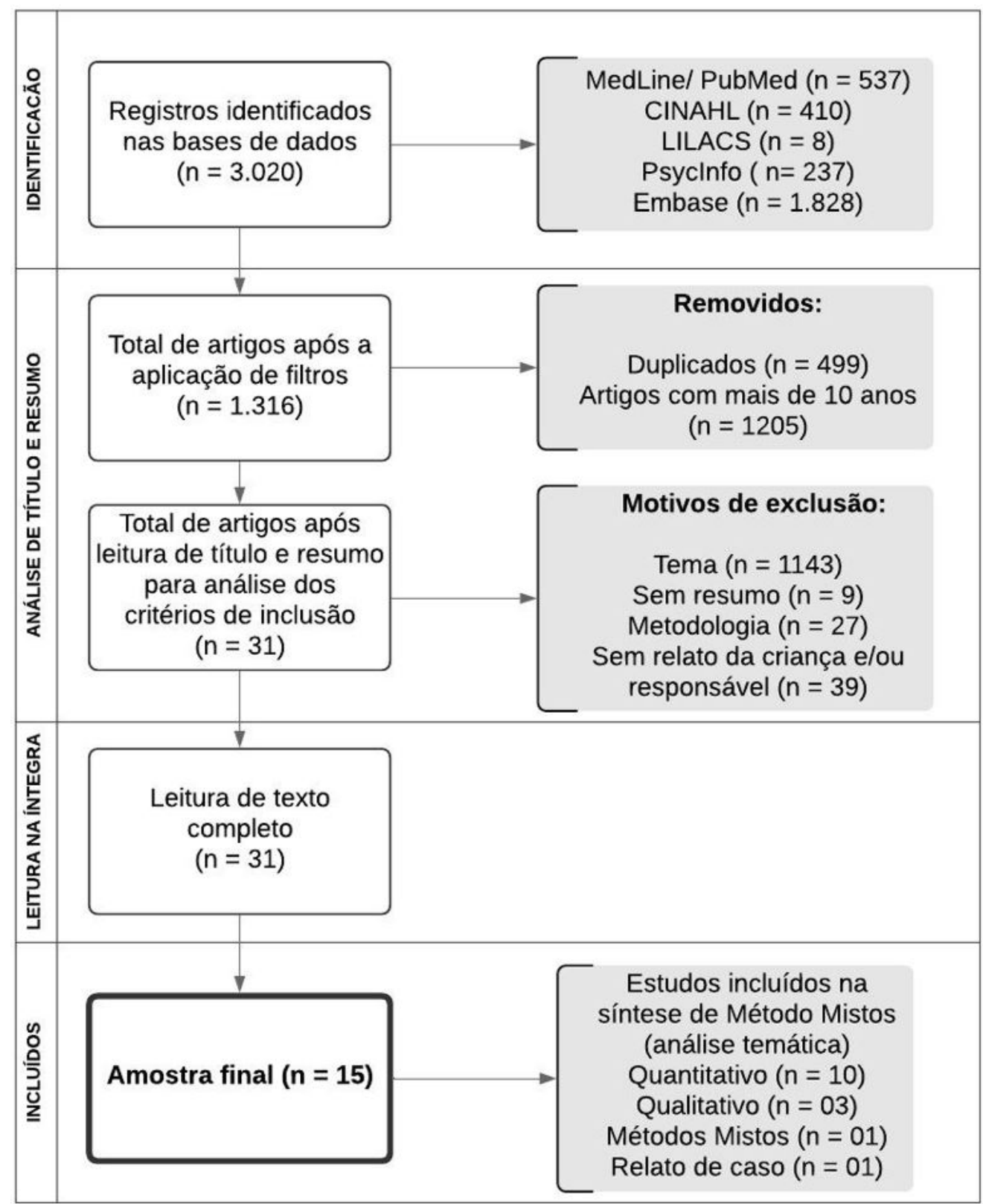

Figura 2. Fluxograma da busca nas bases de dados, adaptação segundo as recomendações PRISMA ${ }^{9}$ \& ENTREQ. ${ }^{10}$ Brasília/DF, Brasil, 2019. 
critérios, sendo distribuídos quatro critérios para cada tipo metodológico. A pontuação final da avaliação foi obtida a partir do percentual de conformidade de cada estudo nos critérios determinados pela ferramenta, estudos que obtivessem um percentual de $0 \%$ seriam exclusos da amostra.

A Extração e síntese dos dados foi realizada por meio de uma matriz de síntese, descrita em uma planilha de Excel. Foram colhidos dados como: ano de publicação, título do artigo, autores, desenho do estudo, características da amostra, método de coleta de dados, principais resultados, sinais, sintomas e problemas relatados/ reportados no estudo. Parte desses dados estão representados no Quadro $1^{11-25}$.

Tal matriz de síntese permitiu que tanto os dados quantitativos quanto qualitativos fossem analisados independentemente (braços quantitativo e qualitativo) e, após, foram integrados e sintetizados de forma combinada. ${ }^{26}$ Para isso, o PRISMA (Preferred Reporting Items for Systematic Review and Meta-Analysis) ${ }^{9}$ e o ENTREQ ${ }^{10}$ (Standards for reporting qualitative research: a synthesis of recommendations) foram adotados de forma combinada e adaptada no sentido de garantir que todos os aspectos da revisão de métodos mistos fossem avaliados.

Os dados compilados foram então analisados por meio de análise temática descrito por Braun \& Clarke, ${ }^{27}$ sendo organizados e apresentados em categorias temáticas obtidas a partir das seguintes etapas de análise: 1) familiarização dos dados (resultados dos estudos que compuseram a amostra e se relacionavam com a pergunta de pesquisa); 2) geração de códigos iniciais; 3 ) busca por temas; 4) revisão dos temas; 5) definição e titulação dos temas; 6) produção do relatório.

Quadro 1. Amostra final de artigos. Brasília/DF, Brasil, 2019.

\begin{tabular}{|c|c|c|c|c|c|}
\hline Estudo & Autores & Ano & Título & $\begin{array}{l}\text { Desenho do } \\
\text { estudo }\end{array}$ & $\begin{array}{l}\text { Avaliação } \\
\text { MMAT }\end{array}$ \\
\hline E1 & Kaugars et al. ${ }^{11}$ & 2010 & $\begin{array}{l}\text { Families' perspectives on the effect of constipation and } \\
\text { fecal incontinence on quality of life }\end{array}$ & Método Misto & $92 \%$ \\
\hline E2 & Stein et al..$^{12}$ & 2010 & An 8-year-old boy with treatment-resistant encopresis & Relato de caso & $25 \%$ \\
\hline E3 & Rajindrajith et al. ${ }^{13}$ & 2013 & $\begin{array}{l}\text { Quality of life and somatic symptoms in children with } \\
\text { constipation: a school-based study }\end{array}$ & Caso controle & $100 \%$ \\
\hline E4 & Dehghani et al. ${ }^{14}$ & 2013 & $\begin{array}{l}\text { Urinary tract infection and enuresis in children with } \\
\text { chronic functional constipation }\end{array}$ & $\begin{array}{l}\text { Estudo } \\
\text { transversal }\end{array}$ & $75 \%$ \\
\hline E5 & Cederblad et al. ${ }^{15}$ & 2014 & $\begin{array}{l}\text { "Nobody asked us if we needed help": Swedish parents } \\
\text { experiences of enuresis }\end{array}$ & Qualitativo & $100 \%$ \\
\hline E6 & Dehghani et al. ${ }^{16}$ & 2015 & $\begin{array}{l}\text { Clinical manifestations among children with chronic } \\
\text { functional constipation }\end{array}$ & $\begin{array}{l}\text { Estudo } \\
\text { transversal }\end{array}$ & $75 \%$ \\
\hline E7 & Al-Zaben \& Sehlo ${ }^{17}$ & 2015 & $\begin{array}{l}\text { Punishment for bedwetting is associated with child } \\
\text { depression and reduced quality of life }\end{array}$ & Caso controle & $100 \%$ \\
\hline E8 & Tai et al. ${ }^{18}$ & 2015 & $\begin{array}{l}\text { Parents have different perceptions of bed-wetting than } \\
\text { children from six to } 15 \text { years of age }\end{array}$ & Caso controle & $75 \%$ \\
\hline E9 & Akyüz et al. ${ }^{19}$ & 2016 & $\begin{array}{l}\text { Evaluation of behavioral problems in patients with } \\
\text { monosymptomatic nocturnal enuresis: a prospective } \\
\text { controlled trial }\end{array}$ & Caso controle & $75 \%$ \\
\hline E10 & Joinson et al. ${ }^{20}$ & 2016 & $\begin{array}{l}\text { Early childhood psychological factors and risk for } \\
\text { bedwetting at school age in a UK cohort }\end{array}$ & $\begin{array}{l}\text { Coorte } \\
\text { prospectiva }\end{array}$ & $100 \%$ \\
\hline E11 & Olaru et al. ${ }^{21}$ & 2016 & $\begin{array}{l}\text { Chronic Functional Constipation and Encopresis } \\
\text { in Children in Relationship with the Psychosocial } \\
\text { Environment }\end{array}$ & $\begin{array}{l}\text { Coorte } \\
\text { prospectiva }\end{array}$ & $75 \%$ \\
\hline E12 & Sarici et al..$^{22}$ & 2016 & $\begin{array}{l}\text { Prevalence of nocturnal enuresis and its influence on } \\
\text { quality of life in school-aged children }\end{array}$ & $\begin{array}{l}\text { Estudo } \\
\text { transversal }\end{array}$ & $100 \%$ \\
\hline E13 & Grzeda et al. ${ }^{23}$ & 2017 & $\begin{array}{l}\text { Effects of urinary incontinence on psychosocial } \\
\text { outcomes in adolescence }\end{array}$ & $\begin{array}{l}\text { Coorte } \\
\text { prospectiva }\end{array}$ & $100 \%$ \\
\hline E14 & Jönson Ring et al. ${ }^{24}$ & 2017 & $\begin{array}{l}\text { Nocturnal enuresis impaired children's quality of life } \\
\text { and friendships }\end{array}$ & $\begin{array}{l}\text { Estudo } \\
\text { transversal }\end{array}$ & $75 \%$ \\
\hline E15 & Saarikoski et al. ${ }^{25}$ & 2018 & $\begin{array}{l}\text { Voiding school as a treatment of daytime incontinence } \\
\text { or enuresis: Children's experiences of the intervention }\end{array}$ & Qualitativo & $100 \%$ \\
\hline
\end{tabular}




\section{RESULTADOS}

A amostra final desta revisão foi composta de 15 artigos descritos a seguir no Quadro 1. A análise temática de seus resultados principais permitiu a organização em três categorias temáticas principais: "Sinais e sintomas associados à condições urinárias e intestinais", "Impacto psicossocial e emocional dos sintomas urinários e intestinais na infância" e "A relação da criança com sintomas urinários e intestinais e a escola".

\section{Sinais e sintomas associados às condições urinárias e intestinais}

De maneira geral, as crianças e adolescentes investigados nesses estudos tinham sintomas urinários ou intestinais de forma isolada. Os estudos também se restringiram na investigação dos seguintes sintomas: incontinência urinária diurna e noturna, constipação intestinal funcional (CIF) e a incontinência fecal (encoprese). Portanto, evidenciou-se uma lacuna no que tange aos demais sintomas urinários (ex.: aumento da frequência urinária, manobras de contenção, retenção urinária, entre outros) e a concomitância de sintomas urinários e intestinais.

Apesar dos principais sinais e sintomas urinários e intestinais estarem bem definidos no documento normativo de nomenclatura da ICCS, alguns estudos dessa revisão apontaram outras manifestações clínicas que tiveram um impacto significativo para a criança.

No estudo E6, ${ }^{16}$ crianças com CIF, além dos sintomas descritos nos critérios de Roma para diagnóstico de CIF, também apresentaram anorexia (38,3\%), dor abdominal (58,1\%), eritema perianal $(13,1 \%)$, sangue vivo nas fezes $(8,1 \%)$ e fissura anal $(7,2 \%)$. Adicionalmente, o estudo E3, ${ }^{13}$ ao avaliar Qualidade de Vida (QV), identificou uma correlação estatisticamente significativa entre dor abdominal e menores escores na escala Pediatric Quality of Life Inventory.

Ao investigar sintomas urinários e intestinais na população pediátrica, o profissional deve investigar outras complicações como Infecções do Trato Urinário (ITU), por exemplo. O estudo E4 ${ }^{14}$ avaliou a frequência de ITU e enurese noturna em crianças com CIF e identificou a ocorrência concomitante de piúria (8,3\%), hematúria $(4,2 \%)$, entre outros sintomas já descritos pela ICCS.

\section{Impacto psicossocial e emocional dos sintomas urinários e intestinais na infância}

Os resultados desta revisão sistemática de métodos mistos demonstraram que a dimensão psicossocial e emocional foi significativamente afetada pela ocorrência de sintomas urinários e intestinais. Os sentimentos reportados pelas crianças e suas famílias, em geral, foram negativos, como demonstrado no estudo $\mathrm{E} 11,{ }^{21}$ onde crianças com CIF apresentaram privação afetiva $(52,63 \%)$, ansiedade $(38,59 \%)$, dificuldade de ajuste social $(22,81 \%)$, timidez $(21,05 \%)$ e baixa tolerância à frustração $(19,29 \%)$.

O Quadro 2 permite visualizar um compilado dos principais sentimentos/emoções descritos pelos estudos que compõem a amostra final desta revisão sistemática de métodos mistos.
A enurese noturna e a incontinência urinária diurna foram descritas pelas crianças como um problema constrangedor, o qual precisam esconder a todo custo. Esse achado mostra como os sintomas urinários limitam a vida social e impactam na autoimagem e autoestima das crianças, como constatados nos estudos E8, ${ }^{18} \mathrm{E} 13,{ }^{23} \mathrm{E} 14^{24}$ e E15. ${ }^{25}$

Adicionalmente, o estudo $\mathrm{E}_{13^{23}}$ identifica sintomas depressivos em $6,8 \%$ das crianças com incontinência urinária diurna e enurese noturna, sendo mais comum em meninas (8,7\%) do que em meninos (4,9\%). Foi verificado que as meninas sentem um impacto maior no quesito autoimagem, já os meninos têm uma percepção pior da escola e mais problemas de relacionamento entre os pares. Além disso, segundo o estudo E5, ${ }^{15}$ à medida que as crianças crescem, o sintoma urinário começa a ser visto como um fardo e os níveis de intolerância parental aumentam, bem como uma maior incidência de complicações advindas dos sintomas urinários e intestinais não manejados adequadamente, tais como hospitalizações, antibioticoterapias e crises álgicas.

Em contrapartida a essas percepções negativas, o $E 1^{11}$ constatou que $75 \%$ das crianças com idade entre 7 a 13 anos não enxergaram a CIF como um problema ou se sentiram pouco incomodadas por esse sintoma. As crianças participantes desse estudo $\mathrm{E} 1{ }^{11}$ citaram emoções como: calma, felicidade, nervosismo e preocupação, sendo a primeira a mais prevalente. Ainda na percepção da criança, o estudo aponta que $37,5 \%$ se incomodaram com o procedimento de enema, $62,5 \%$ se preocuparam com o desempenho escolar e $85,5 \%$ consideraram que os acidentes fecais possuíam odor desagradável.

$\mathrm{O}$ estudo $\mathrm{E} 1^{11}$ apontou ainda a percepção da família sobre a CIF na criança, relatando sentimentos/emoções como nervosismo, tristeza e preocupação. Do total das famílias estudadas, 37,5\% relataram se incomodar com a ausência de melhora da condição da criança, $85,7 \%$ com o odor oriundo das perdas e $50 \%$ consideravam o tratamento como algo traumático, uma vez que era recorrente o choro da criança na realização de procedimentos invasivos como o enema. Outro impacto relatado diz respeito à dimensão financeira, as famílias sinalizaram aumento dos custos com roupas extras e lavanderia devido às perdas fecais da criança.

A família também relatou implicações psicossociais negativas causadas pelo sintoma urinário, como demonstrado no estudo E5. ${ }^{15}$ Segundo relato de pais de crianças com enurese noturna, ter um filho nesta condição pode ser estressante e vergonhoso. Embora os pais tentavam não culpabilizar seu filho, a intolerância parental aumentava à medida que os sintomas urinários persistiam e na percepção dos pais, a enurese era percebida como algo socialmente estigmatizante e incapacitante.

Outro fator relacionado à manutenção de sintomas urinários e intestinais é a punição parental. O estudo $E 7{ }^{17}$ avaliou a relação da punição parental frente aos episódios de enurese e sua correlação com a depressão, considerando a percepção dos pais. Foi evidenciado que ao apresentarem episódios de perda urinária durante a noite, as crianças eram punidas e por consequência apresentaram sintomas depressivos significativamente maiores 
Quadro 2. Síntese dos principais sentimentos/emoções descritos pelos estudos incluídos na revisão sistemática de métodos mistos. Brasília/DF, Brasil, 2019.

\begin{tabular}{|c|c|c|c|}
\hline Representação gráfica & $\begin{array}{l}\text { Sentimento/ } \\
\text { Emoção }\end{array}$ & Resultado do Estudo & $\begin{array}{l}\text { Perspectiva do } \\
\text { Respondente }\end{array}$ \\
\hline & $\begin{array}{l}\text { Nervosismo }{ }^{\mathrm{E} 1} \\
\text { Medo }{ }^{\mathrm{E}}\end{array}$ & $\begin{array}{l}\text { O nervosismo e o medo apareceram em } 75 \% \text { dos } \\
\text { relatos de crianças com CIF, quando a questão era } \\
\text { relacionada as idas ao médico. }\end{array}$ & Criança e Família E1 \\
\hline & Preocupação ${ }^{\mathrm{E2} \text {, E1 }}$ & $\begin{array}{l}\text { No estudo E1, 62,5\% das crianças relataram } \\
\text { preocupação em relação ao desempenho na escola. } \\
\text { Os pais reportaram preocupação com a condição } \\
\text { da criança e apresentaram comportamentos mais } \\
\text { tolerantes e protetivos frente à criança. No estudo } \\
\text { E2, a criança vivenciou uma experiência de separação } \\
\text { da mãe durante o processo de desfralde, o que pode } \\
\text { ter contribuído para o início da encoprese. Segundo } \\
\text { relato dos profissionais que assistiram o caso, a criança } \\
\text { demonstrava preocupação com as perdas fecais e } \\
\text { comportamento retentivo. }\end{array}$ & $\begin{array}{l}\text { Família E1 } \\
\text { Criança E1 } \\
\text { Profissionais E2 }\end{array}$ \\
\hline & $\begin{array}{l}\text { VergonhaE5/ } \\
\text { Timidez }^{\text {E11,E15 }}\end{array}$ & $\begin{array}{l}\text { No estudo E5, a família percebia a vergonha da criança } \\
\text { e também se sentia envergonhada, relatou medo } \\
\text { da comunidade saber e fofocar sobre a condição da } \\
\text { criança. No estudo E15, os pais sentiam que deviam } \\
\text { proteger a criança das situações constrangedoras. } \\
\text { Assim, evitavam falar sobre o sintoma urinário na } \\
\text { frente de desconhecidos para preservar a privacidade } \\
\text { da criança. Também se preocupavam com o futuro da } \\
\text { criança em relação a autoestima. }\end{array}$ & $\begin{array}{l}\text { Família E5 } \\
\text { Criança E11,E15 }\end{array}$ \\
\hline
\end{tabular}

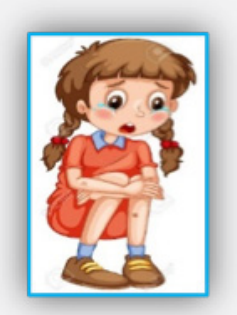

As crianças com encoprese apresentavam ansiedade

Ansiedade ${ }^{\mathrm{E} 11}$ $(38,59 \%)$ e os episódios de encoprese influenciaram no

Criança ${ }^{\mathrm{E} 11}$ número de faltas na escola.

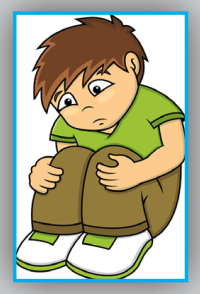

No estudo E7 foi evidenciado que $33,8 \%$ das crianças estudadas sofriam punições dos pais em decorrência da enurese e que quanto maior a frequência dos

Sintomas Depressivos $^{\mathrm{E7}, \mathrm{E} 13}$ episódios de enurese, maior era o tempo de punição o que resultou em impacto na qualidade de vida Família E7 e aumento dos sintomas depressivos. No estudo E13, 6,8\% dos adolescentes apresentaram sintomas depressivos e impacto negativo na autoimagem.

Fonte das imagens: https://pt.dreamstime.com/ 
e mais severos se comparadas às crianças que não sofriam punição. Ainda neste estudo, $33,8 \%$ das crianças sofriam punições por parte dos pais, sendo mais comum a medida punitiva verbal associada à lesão física $(31,8 \%)$. Essas crianças, quando comparadas com as que não sofriam punições tiveram pior performance escolar, aumento dos episódios de enurese e impacto negativo na qualidade de vida.

\section{A relação da criança e adolescente com sintomas urinários e intestinais e a escola}

A escola foi pontuada como um espaço socialmente hostil e estressante para a criança e o adolescente com sintomas urinários e intestinais. $O$ adolescente tem receio de que seu problema urinário seja descoberto por colegas e se torne alvo de bullying. Assim, a criança esconde de seus pares seu problemae teme a ocorrência de perdas urinárias durante o período escolar, como descrito no estudo E15. ${ }^{25}$

A relação entre os pares na escola foi também permeada por vitimizações. Estudo realizado com pais de adolescentes com incontinência urinária no Reino Unido (E13) mostrou que cerca de $17,8 \%$ passaram por essas situações. ${ }^{23}$ Dados do estudo E14 ${ }^{24}$ com crianças suecas mostraram também que, ao se comparar os sexos, os meninos reportaram maior impacto do que as meninas e, quanto mais velhos, também essa repercussão foi maior.

Outro aspecto trazido pela revisão foi quanto ao rendimento escolar da criança/ adolescente. O estudo E11, ${ }^{21}$ uma coorte prospectiva com 57 pacientes incontinentes fecais de 6 a 15 anos, identificou situações de abandono escolar, reprovação de ano letivo e numerosas faltas escolares. Tais dados que se assemelham aos achados de baixo desempenho escolar em crianças com enurese noturna do estudo E12, ${ }^{22}$ justificando a preocupação de pais no que diz respeito ao impacto no desempenho escolar das crianças com CIF e encoprese, descritas pelo estudo E11. ${ }^{21}$ Além desses fatores, foi identificado no estudo $E 7^{17}$ que crianças que sofrem punição por conta dos episódios de perdas urinárias noturnas apresentavam pior performance escolar, quando comparados com crianças que não passam por tais penalidades.

Em suma, a escola tem sido alvo de uma percepção ruim por parte de crianças e adolescentes com sintomas urinários e intestinais e, em certos casos, essa percepção se aplica também aos professores (E13). ${ }^{23} \mathrm{O}$ estudo $E 8^{18}$ por meio da escala Teenage Self-Concept Scale conseguiu captar que crianças com enurese tinham menor autoconceito escolar, e esse índice era pior nas crianças maiores de 11 anos.

\section{DISCUSSÃO}

Esta revisão de métodos mistos trouxe uma síntese quanti-qualitativa sobre como as crianças e suas famílias percebem e lidam com os sintomas urinários e intestinais, bem como as experiências vividas. No entanto, houve uma predominância de estudos quantitativos (11 pesquisas), fato que impossibilitou a captação extensa de dados subjetivos relacionados à temática. Os estudos conseguiram discutir os aspectos psicossociais por meio de instrumentos validados, como os que mensuram QV. .1113 $^{11}$
Dessa forma, existe uma lacuna na literatura de estudos que explorem mais o relato do sujeito (criança e sua família) no que diz respeito a experiência de sintomas urinários e intestinais.

Os sintomas descritos com maior frequência nos estudos foram incontinência urinária diurna, enurese noturna, CIF e encoprese isolados e não concomitantes. Apontando assim, uma carência de trabalhos que discutam os demais sintomas do trato urinário inferior como aumento/diminuição da frequência urinária, manobras de contenção, urgência miccional, ${ }^{1}$ bem como a associação desses com os sintomas intestinais.

Em contrapartida, outro sintoma pouco discutido nos documentos de sociedades internacionais foi abordado. A dor abdominal nas crianças com CIF foi associada com menor índice $\mathrm{QV},{ }^{16}$ sendo este um sintoma que modifica a experiência da criança com CIF. Tal dado foi corroborado por outros estudos descritos na literatura, dentre eles um estudo realizado com crianças escolares sem doença específica ${ }^{28}$ e outro estudo que investigou crianças com doença de Crohn.${ }^{29} \mathrm{Em}$ ambos os casos, a dor impactou negativamente nas diversas dimensões que compõem o conceito de QV.

No que tange às repercussões de cunho psicossocial e emocional em crianças com sintomas urinários e/ou intestinais, a literatura tem mostrado que pacientes pediátricos são particularmente propensos a desenvolver distúrbios emocionais. ${ }^{30}$ Os sintomas urinários e/ou intestinais podem influenciar de forma negativa aspectos da rotina da criança, sobretudo aqueles inerentes ao contexto familiar e escolar. Desse modo se faz necessário monitorar os efeitos psicossociais durante o acompanhamento interdisciplinar da criança. ${ }^{31}$

Frente aos sentimentos vivenciados em decorrência dos sintomas urinários e/ou intestinais, os resultados encontrados nesta revisão sistemática estão de acordo com o demonstrado na literatura que refere que, a autoestima baixa, insegurança, ansiedade e isolamento. Também são os mesmos sintomas e emoções reportados por crianças que vivenciam a DVI e que essa experiência negativa resulta em impacto na qualidade de vida da criança e sua família. ${ }^{30,32}$

Considerando a ótica dos familiares, foi evidenciado que a experiência com os sintomas urinários e/ou intestinais da criança Ihes geravam sentimentos como nervosismo, medo, vergonha, estresse e preocupação. Esse achado corrobora com o referido no estudo de Mota et al., ${ }^{33}$ onde aponta que a família refere sentimento de preocupação frente ao sintoma urinário de seu filho. Nesta revisão, o sentimento de ansiedade foi vivenciado pela criança, mas Tanriverdi et al. ${ }^{34}$ demonstraram que os pais também apresentam ansiedade diante do sintoma urinário da criança.

O uso da punição pelos responsáveis como recurso para corrigir as crianças com sintomas urinários também foi identificado nesta revisão sistemática. Esse achado de igual forma foi mencionado no estudo de Sapi et al..$^{35}$ que avaliou a violência em crianças com enurese. Não se tem clareza do impacto a longo prazo desse tipo de violência com a criança, todavia, cabe ao profissional de saúde identificar esses casos, 
desmistificar e desencorajar este tipo de conduta por parte dos cuidadores/família.

Em oposição ao apontado em outros estudos, algumas crianças não percebem os sintomas urinários como problema. Tal aspecto poderia explicar os casos em que a criança não se sente motivada para modificações propostas pelo profissional de saúde nos programas de uroterapia, ${ }^{36}$ considerando que para ela os sintomas urinários não são uma queixa e/ou problema, mas sim de seus responsáveis.

Dentre os fatores contribuintes para o início e a manutenção de problemas de eliminação estão os eventos traumáticos e estressores, como por exemplo a separação da mãe e criança durante o processo de desfralde, como descrito no estudo E2. ${ }^{14}$ Esse estudo descreveu o caso de uma criança de 8 anos com uma longa história de encoprese e enurese. Vale destacar que famílias que não acompanham o treinamento esfincteriano e que desconhecem os hábitos de eliminação vesical e intestinal da criança contribuem para o desenvolvimento de problemas de eliminação. ${ }^{12}$

A literatura já tem apontado algumas dificuldades que a criança pode encontrar no ambiente escolar, como a infraestrutura inadequada de banheiros e a não permissão para o uso da toalete durante as aulas. ${ }^{37,38}$ Além disso, a criança pode sofrer com o medo de escapes neste ambiente. Estudo brasileiro com crianças escolares mostrou que $20 \%$ dos entrevistados $(n=17)$ afirmaram já ter tido experiência prévia de perda urinária na escola, ${ }^{37}$ assim, este é um evento relativamente comum no ambiente escolar e pode estar relacionado com as dificuldades citadas anteriormente.

Os estudos da amostra apontaram ainda que as crianças com esses sintomas têm apresentado baixo rendimento escolar, medo relacionado ao bullying e de maneira geral uma relação ruim com seus pares. ${ }^{21,22}$ Elementos que tornam ainda mais difícil para a criança a experiência de vida e devem ser pontos de avaliação pelo profissional de saúde.

Dessa forma, a avaliação constante desse ambiente social é essencial, e pode ser realizada com auxílio de instrumentos validados de qualidade de vida que já vislumbra a escola com um espaço fundamental para a compressão do eixo social da criança, como foi realizado, por exemplo, em estudo no Sri Lanka (E3) com crianças que apresentavam $\mathrm{CIF}^{13} \mathrm{Cabe}$, portanto, à equipe de saúde o trabalho em rede com a escola a fim de minimizar tais implicações psicossociais, esclarecer dúvidas da comunidade escolar e promover um ambiente saudável para a criança acometida por sintomas urinários e/ou intestinais.

\section{CONCLUSÃO E CONSIDERAÇÕES PARA A PRÁTICA}

Com esta revisão sistemática de métodos mistos foi possível alcançar parcialmente o objetivo traçado devido ao perfil metodológico dos estudos da amostra e os sintomas investigados. Dessa forma, o mapeamento da ocorrência de sintomas urinários e intestinais ficou limitado aos sintomas de incontinência diurna, enurese noturna, CIF e encoprese.
E as experiências vividas pelas crianças e suas famílias foram igualmente restritas, tendo em vista que fizeram parte da amostra apenas dois estudos qualitativos e um de método misto. Isto posto, se faz necessário a realização de pesquisas futuras que investiguem os demais sintomas urinários e intestinais e em uma perspectiva que favoreça o relato da criança e sua família.

A partir dos dados obtidos na revisão, identifica-se que a criança com esses sintomas apresenta impacto nas dimensões emocional e social. Foram descritos sentimentos de nervosismo, ansiedade, sintomas depressivos, vergonha, baixa tolerância à frustração; bem como problemas com imagem corporal e dificuldade de relacionamento com os colegas e outros. A escola, dentro da dimensão social, foi apontada como o ambiente mais impactado pelos sintomas urinários e/ou intestinais. Nela a criança, em diversos casos, apresenta baixo rendimento escolar, medo relacionado ao bullying e de maneira geral uma relação ruim com seus colegas.

Em suma, essa revisão sugere a necessidade de pesquisas futuras no sentido de melhorar a compreensão das experiências psicossociais dos sintomas urinários e intestinais sobre crianças/adolescentes e suas famílias durante toda a vida. Além de despertar os profissionais de saúde e da educação para a atenção especial da experiência destes sintomas em crianças e suas famílias. Dessa forma, uma estratégia possível é a viabilização de momentos de diálogo e verbalização desses problemas pela criança e seus familiares, a fim de amenizar os impactos por eles trazidos.

\section{CONTRIBUIÇÕES DOS AUTORES}

Concepção e desenho do estudo. Extração de dados, análise e interpretação dos dados. Discussão dos resultados. Redação e revisão crítica do manuscrito. Aprovação da versão final do artigo. Responsabilidade por todos os aspectos do conteúdo e a integridade do artigo publicado: Cristiane Feitosa Salviano. Extração de dados. Discussão dos resultados. Redação e revisão crítica do manuscrito. Aprovação da versão final do artigo. Responsabilidade por todos os aspectos do conteúdo e a integridade do artigo publicado: Priscilla Lemos Gomes. Concepção e desenho do estudo. Interpretação dos dados. Discussão dos resultados. Redação e revisão crítica do manuscrito. Aprovação da versão final do artigo. Responsabilidade por todos os aspectos do conteúdo e a integridade do artigo publicado: Gisele Martins.

\section{EDITOR ASSOCIADO}

\author{
Aline Cristiane Okido
}

\section{REFERÊNCIAS}

1. Austin PF, Bauer SB, Bower W, Chase J, Franco I, Hoebeke P et al. The standardization of terminology of lower urinary tract function in children and adolescents: update report from the standardization committee of the International Children's Continence Society. Neurourol Urodyn. 2016;35(4):471-81. http://dx.doi.org/10.1002/nau.22751.PMid:25772695.

2. Van Summeren J, Holtman GA, van Ommeren SC, Kollen BJ, Dekker JH, Berger MY. Bladder symptoms in children with functional constipation: a 
systematic review. J Pediatr Gastroenterol Nutr.2018;67(5):552-60. http:// dx.doi.org/10.1097/MPG.0000000000002138. PMid:30212423.

3. FarhatW, Bägli DJ, Capolicchio G, O'Reilly S, Merguerian PA, Khoury A etal. The dysfunctional voiding scoring system: quantitative standardization of dysfunctional voiding symptoms in children. J Urol. 2000;164(3):1011-5. http://dx.doi.org/10.1016/S0022-5347(05)67239-4. PMid:10958730.

4. Santos JD, Lopes RI, Koyle MA. Bladder and bowel dysfunction in children: an update on the diagnosis and treatment of a common, but underdiagnosed pediatric problem. Can Urol Assoc J. 2017;11(1-2):S64-72. http://dx.doi. org/10.5489/cuaj.4411. PMid:28265323.

5. Lenz E, Pugh LC, Milligan RA, Gift A, Suppe F. The middle-range theory of unpleasant symptoms: an update. ANS Adv Nurs Sci. 1997;19(3):14-27. http://dx.doi.org/10.1097/00012272-199703000-00003. PMid:9055027.

6. Martins G, Minuk J, Varghese A, Dave S, Williams K, Farhat WA. Nonbiological determinants of paediatric bladder bowel dysfunction: a pilot study. J Pediatr Urol. 2016;12(2):109.e1. http://dx.doi.org/10.1016/j. jpurol.2015.09.006. PMid:26586295.

7. Assis GM, Silva CPC, Martins G. Urotherapy in the treatment of children and adolescents with bladder and bowel dysfunction: a systematic review. J Pediatr.2019;95(6):628-41.http://dx.doi.org/10.1016/j.jped.2019.02.007.

8. Pluye P, Hong QN. Combining the power of stories and the power of numbers: mixed methods research and mixed studies reviews. Annu Rev Public Health. 2014;35(1):29-45. http://dx.doi.org/10.1146/annurevpublhealth-032013-182440. PMid:24188053.

9. Liberati A, Altman DG, Tetzlaff J, Mulrow C, Gotzsche PC, loannidis JPA et al. The PRISMA statement for reporting systematic reviews and meta-analyses of studies that evaluate health care interventions: Explanation and elaboration. J Clin Epidemiol. 2009;62(10):e1-34. http:// dx.doi.org/10.1016/j.jclinepi.2009.06.006. PMid:19631507.

10. Tong A, Flemming K, Mclnnes E, Oliver S, Craig J. Enhancing transparency in reporting the synthesis of qualitative research: ENTREQ. BMC Med Res Methodol. 2012;12(1):181. http://dx.doi.org/10.1186/1471-2288-12-181. PMid:23185978.

11. Kaugars AS, Silverman A, Kinservik M, Heinze S, Reinemann L, Sander $M$ et al. Families' perspectives on the effect of constipation and fecal incontinence on quality of life. J Pediatr Gastroenterol Nutr. 2010;51(6):74752. http://dx.doi.org/10.1097/MPG.0b013e3181de0651.PMid:20706148.

12. Stein MT, Benninga MA, Felt BT. An 8-year-old boy with treatment-resistant encopresis. J Dev Behav Pediatr.2010;31(6):513-5. http://dx.doi.org/10.1097/ DBP.0b013e3181e5a464. PMid:20611039.

13. Rajindrajith S, Devanarayana NM, Weerasooriya L, HathagodaW, Benninga MA. Quality of life and somatic symptoms in children with constipation: a school-based study. J Pediatr. 2013;163(4):1069-72.e1. http://dx.doi. org/10.1016/j.jpeds.2013.05.012. PMid:23800401.

14. Dehghani SM, Basiratnia M, Matin M, Hamidpour L, Haghighat M, Imanieh $\mathrm{MH}$. Urinary tract infection and enuresis in children with chronic functional constipation. Iran J Kidney Dis. 2013;7(5):363-6. PMid:24072148.

15. Cederblad M, Neveus T, Ahman A, Österlund Efraimsson E, Sarkadi A. "Nobody asked us if we needed help": swedish parents experiences of enuresis. J Pediatr Urol. 2014;10(1):74-9. http://dx.doi.org/10.1016/j. jpurol.2013.06.006. PMid:23849996.

16. Dehghani SM, Kulouee N, Honar N, Imanieh MH, Haghighat M, Javaherizadeh $\mathrm{H}$. Clinical manifestations among children with chronic functional constipation. Middle East J Dig Dis. 2015;7(1):31-5. http://dx.doi. org/10.15171/mejdd.2017.87. PMid:25628851.

17. Al-Zaben FN, Sehlo MG. Punishment for bedwetting is associated with child depression and reduced quality of life. Child Abuse Negl. 2015;43:22-9. http://dx.doi.org/10.1016/j.chiabu.2014.11.007. PMid:25435105.

18. Tai TT, Tai BT, Chang YJ, Huang KH. Parents have different perceptions of bed-wetting than children from six to 15 years of age. Acta Paediatr. 2015;104(10):e466-72. http://dx.doi.org/10.1111/apa.13101.PMid:26119996.

19. Akyüz M, Koca O, Karaman B, Özcan ZY, Öztürk Mi, Kutluhan MA et al. Evaluation of behavioral problems in patients with monosymptomatic nocturnal enuresis: a prospective controlled trial. Turk J Med Sci. 2016;46(3):807-11. http://dx.doi.org/10.3906/sag-1502-90. PMid:27513260.

20. Joinson C, Sullivan S, von Gontard A, Heron J. Early childhood psychological factors and risk for bedwetting at school age in a UK cohort. Eur Child
Adolesc Psychiatry.2016;25(5):519-28. http://dx.doi.org/10.1007/s00787015-0756-7. PMid:26294078.

21. Olaru C, Diaconescu S, Trandafir L, Gimiga N, Olaru RA, Stefanescu G et al Chronic functional constipation and encopresis in children in relationship with the psychosocial environment. Gastroenterol Res Pract. 2016;2016:7828576 http://dx.doi.org/10.1155/2016/7828576. PMid:27990158.

22. Sarici H, Telli O, Ozgur BC, Demirbas A, Ozgur S, Karagoz MA. Prevalence of nocturnal enuresis and its influence on quality of life in school-aged children. J Pediatr Urol. 2016;12(3):159.e1-6. http://dx.doi.org/10.1016/j. jpurol.2015.11.011. PMid:26778419.

23. Grzeda M, Heron J, von Gontard A, Joinson C. Effects of urinary incontinence on psychosocial outcomes in adolescence. Eur Child Adolesc Psychiatry. 2017;26(6):649-58. http://dx.doi.org/10.1007/s00787-016-0928-0. PMid:27943057.

24. Jönson Ring I, Nevéus T, Markström A, Arnrup K, Bazargani F. Nocturnal enuresis impaired children's quality of life and friendships. Acta Paediatr. 2017;106(5):806-11. PMid:28199734.

25. Saarikoski A, Koppeli R, Salanterä S, Taskinen S, Axelin A. Voiding schoo as a treatment of daytime incontinence or enuresis: children's experiences of the intervention. J Pediatr Urol. 2018;14(1):56.e1-7. http://dx.doi. org/10.1016/j.jpurol.2017.09.009. PMid:29037865.

26. Heyvaert M, Maes B, Onghena P. Mixed methods research synthesis: definition, framework, and potential. Qual Quant. 2013;47(2):659-76. http:// dx.doi.org/10.1007/s11135-011-9538-6.

27. Braun V, ClarkeV.Using thematic analysis in psychology. Qual Res Psychol. 2006;3(2):77-101. http://dx.doi.org/10.1191/1478088706qp063oa.

28. Haraldstad K, Christophersen KA, Helseth S. Health-related quality of life and pain in children and adolescents: a school survey. BMC Pediatr. 2017;17(1):174.http://dx.doi.org/10.1186/s12887-017-0927-4.PMid:28738818.

29. Claar RL, van Tilburg MAL, Abdullah B, Langer S, Sherif D, Whitehead WE et al. Psychological distress and quality of life in pediatric crohn disease: impact of pain and disease state. J Pediatr Gastroenterol Nutr. 2017;65(4):420-4. http://dx.doi.org/10.1097/MPG.0000000000001549. PMid:28945206.

30. Azevedo RVM, Oliveira EA, Vasconcelos MMA, Castro BAC, Pereira FR, Duarte NFV et al. Impact of an interdisciplinary approach in children and adolescents with lower urinary tract dysfunction (LUTD). J Bras Nefrol. 2014;36(4):451-9. http://dx.doi.org/10.5935/0101-2800.20140065. PMid:25517273.

31. Veloso LA, Mello MJ, Ribeiro No JP, Barbosa LN, Silva EJ. Quality of life, cognitive level and school performance in children with functional lower urinary tract dysfunction. J Bras Nefrol. 2016;38(2):234-44. http://dx.doi. org/10.5935/0101-2800.20160033. PMid:27438979.

32. Vasconcelos TB, Cavalcante LIC. Avaliação das atividades de vida diária em crianças: uma revisão da literatura. Rev Ter Ocup Univ Sao Paulo. 2013;24(3):267-72. http://dx.doi.org/10.11606/issn.2238-6149. v24i3p267-272.

33. Mota DM, Barros AJD, Matijasevich A, Santos IS. Prevalence of enuresis and urinary symptoms at age 7 years in the 2004 birth cohort from Pelotas, Brazil. J Pediatr. 2015 Jan/Feb;91(1):52-8. http://dx.doi.org/10.1016/j. jped.2014.04.011. PMid:25193596.

34. Tanrıverdi MH, PalancıY, Yılmaz A, Penbegül N, BezY, Dağgülli M. Effects of enuresis nocturna on parents of affected children: casecontrol study. Pediatr Int. 2014;56(2):254-7. http://dx.doi.org/10.1111/ped.12242. PMid:24467519.

35. Sapi MC, Vasconcelos JS, Silva FG, Damião R, Silva EA. Assessment of domestic violence against children and adolescents with enuresis. J Pediatr. 2009;85(5):433-7. PMid:19830354.

36. Souza BML, Salviano CF, Martins G. Prática avançada de enfermagem em uropediatria: relato de experiência no Distrito Federal. Rev Bras Enferm. 2018 Feb;71(1):223-7. http://dx.doi.org/10.1590/0034-71672016-0654. PMid:29324966.

37. Salviano CF, Martins G. Contexto escolar e hábitos miccionais: estudo transversal com escolares do Distrito Federal. Cienc Cuid Saude. 2016 Apr/ Jun;15(2):235-41. http://dx.doi.org/10.4025/cienccuidsaude.v15i2.28173.

38. Souza BML, Salviano CF, Martins G. Contexto escolar e sintomas de trato urinário inferior: revisão integrativa da literatura. Cogitare Enferm. 2015 Jan/Mar;20(1):198-206. http://dx.doi.org/10.5380/ce.v20i1.37477. 